\title{
Direct referrals from emergency department streaming to ambulatory care: improving same-day emergency care
}

\author{
Authors: Waqas Jarral, ${ }^{A *}$ Bethan Graf, ${ }^{A}$ Sean Harding, ${ }^{A}$ Gareth Roberts ${ }^{A}$ and John Seymour ${ }^{A}$
}

\section{Introduction}

Same-day emergency care is one of the many ways the NHS is working to provide the right care, in the right place, at the right time for patients. It aims to benefit both patients and the healthcare system by reducing waiting times and unnecessary hospital admissions.

In our organisation patients presenting to the emergency department (ED) with common medical conditions like chest pain / shortness of breath (SOB), palpitations, headache, chest infection or deep vein thrombosis / pulmonary embolism were initially triaged in ED streaming. There were delays due to initial assessment by emergency care doctors and subsequent assessment by the on-call medical team.

We developed a pathway of direct referrals from ED streaming to ambulatory care for clinically suitable patients with common conditions like chest pain, palpitations, headache or SOB / chest infection. The aim was to avoid unnecessary hospital admissions and therefore reduce the risk of infections and de-conditioning for patients, improve patient experience by reducing waiting times, improve patient flow and reduce congestion in the ED, and achieve financial benefits and cost savings for the hospital.

\section{Method}

Patients were identified from ED streaming with chest pain, SOB, palpitations and headache and fast tracked to ambulatory care. Exclusion criteria were used to ensure safety.

\section{Results and discussion}

During a 4-day period in September 2019, 33 patients were taken directly from ED streaming. Only two of these patients were admitted, achieving $93.93 \%$ of the patients being discharged safely the same day.

In contrast, during the month of August, 20 patients, specifically with suspected PE, were admitted from the ED. In retrospect, 11 of these patients would have been suitable for the same day emergency care as part of our direct referrals pilot.

One of the critical factors leading to unnecessary admissions was the extended period of time associated with the traditional referral process between the ED and the medical take, especially the

Authors: ${ }^{\text {A}}$ rimley Health NHS Foundation Trust, Frimley, UK; *RCP chief registrar patients who presented later in the afternoon and were reviewed out of hours. The average waiting time for ED during the week of pilot was 5 hours 44 minutes as compared with 49 minutes in ambulatory care during the same time period.

\section{Conclusion}

The new pathway ensures that clinically suitable patients are reviewed earlier through ambulatory care by the medical team, hence avoiding unnecessary hospital admissions. This leads to reduced waiting times and improved patient flow between ED and the medical team.

\section{Conflicts of interest}

None declared. 